\title{
Ewing's sarcoma of the cervix: A case report of an unusual diagnosis in pregnancy treated with surgery, adjuvant VIDE and radiotherapy
}

\author{
ANASTASIOS KYRIAZOGLOU ${ }^{1}$, GEORGIOS TSIRONIS ${ }^{1}$, MICHALIS LIONTOS ${ }^{1}$, \\ ALEXANDRA PAPAKOSTA $^{2}$, LUISA MAHAIRA ${ }^{2}$, NIKOLAOS THOMAKOS ${ }^{3}$, \\ GEORGIOS MORPHOPOULOS $^{4}$, IRENE PAPASPYROU ${ }^{4}$ and ARISTOTELIS BAMIAS ${ }^{1}$
}

${ }^{1}$ Department of Clinical Therapeutics, Alexandra Hospital; ${ }^{2}$ Department of Genetics, Agios Savvas Hospital;

Departments of ${ }^{3}$ Obstetrics and Gynecology and ${ }^{4}$ Pathology, Alexandra Hospital, 11528 Athens, Greece

Received May 7, 2018; Accepted September 27, 2018

DOI: $10.3892 / \mathrm{ol} .2019 .10267$

\begin{abstract}
Ewing's sarcoma of the cervix is a rare entity and presents with considerable challenges in diagnosis and therapy. Herein, we report a case of a cervical Ewing's sarcoma presenting with FIGO stage Ib, diagnosed during the first trimester of the patient's pregnancy. Imaging with CT scans, MRI of her abdomen and PET-CT verified the locoregional extension of the tumor. The diagnosis was confirmed by immunohistochemistry and molecular analysis. Fluorescence in situ hybridization and RT-PCR detected the pathognomonic EWS/FLI fusion gene. Favorable prognostic factors regarding the stage, clinocopathological and molecular characteristics of the tumor are also described. Due to the rarity of the disease, at present, there is no universal consensus on the optimal therapeutic approach. The literature has been reviewed and the therapeutic schemes and available clinical data have been discussed. The patient presented in this case report was treated aggressively with tri-modality therapy and underwent radical hysterectomy followed by adjuvant chemotherapy with Vincristine-Ifosfamide-Doxorubicin-Etoposide and radiotherapy. The patient remains free of this disease 42 months following the diagnosis of her tumor.
\end{abstract}

\section{Introduction}

Cervical sarcomas are rare and constitute $<1 \%$ of all cervical malignancies (1). A specific subtype, Ewing sarcoma of the cervix, is an extremely rare tumor and for this reason

Correspondence to: Dr Anastasios Kyriazoglou, Department of Clinical Therapeutics, Alexandra Hospital, Vasilisis Sofias 80, 11528 Athens, Greece

E-mail: tassoskyr@gmail.com

Key words: cervical Ewing's sarcoma, adjuvant chemotherapy, VIDE, radiotherapy, pregnancy particularly challenging regarding the choice of optimal therapeutic strategy (2).

Ewing sarcoma is a mesenchymal malignancy with specific genetic and immunohistochemical characteristics, mainly affecting the bones. However, 20-30\% of Ewing sarcomas arise from an extraosseous site (3). Extraosseous Ewing sarcomas affect patients of any age. The diagnosis of Ewing sarcomas of the female genital tract is extremely rare. The identification of reciprocal translocation $\mathrm{t}(11 ; 22)$ and the subsequent formation of the fusion gene EWS/FLI are pathognomonic for the diagnosis of this tumor (4).

Several different therapeutic modalities have been applied to the few reported cases in the literature (5-17). ESMO and NCCN guidelines recommend to treat these tumors like uterine sarcomas $(18,19)$. Nevertheless, there is no universal consensus on the therapeutic approach of cervical Ewing sarcomas. The coexistence of pregnancy makes the therapeutic strategy challenging. Only 6 cases with extraosseous Ewing sarcomas during pregnancy have been reported $(13,20)$.

\section{Case report}

A 38 year old woman referred to our hospital in the first trimester of her pregnancy (9th week) due to a tumor mass in her cervix, as an incidental finding during her scheduled first trimester abdominal ultrasound. Pelvic examination revealed that the vulva and the vagina were normal. However, the cervix was enlarged with smooth surface without necrotic lesions. Bimanual examination revealed a large cervical mass measuring $8 \mathrm{~cm}$ in diameter. The size of the uterus was slightly enlarged. There was no extension of the lesion into the vagina, parametria or adjacent organs. MRI of her abdomen revealed a $8 \times 7.6 \mathrm{~cm}$ tumor of the cervix (Fig. 1). PET-CT verified the locoregional extension of the tumor without any indications of metastatic sites (Fig. 1). Tumor biopsy was performed during termination of her pregnancy. The patient was already the mother of 2 children and decided to end her pregnancy. Histopathologic report of tissue specimen favored the diagnosis of Ewing's sarcoma/PNET. 

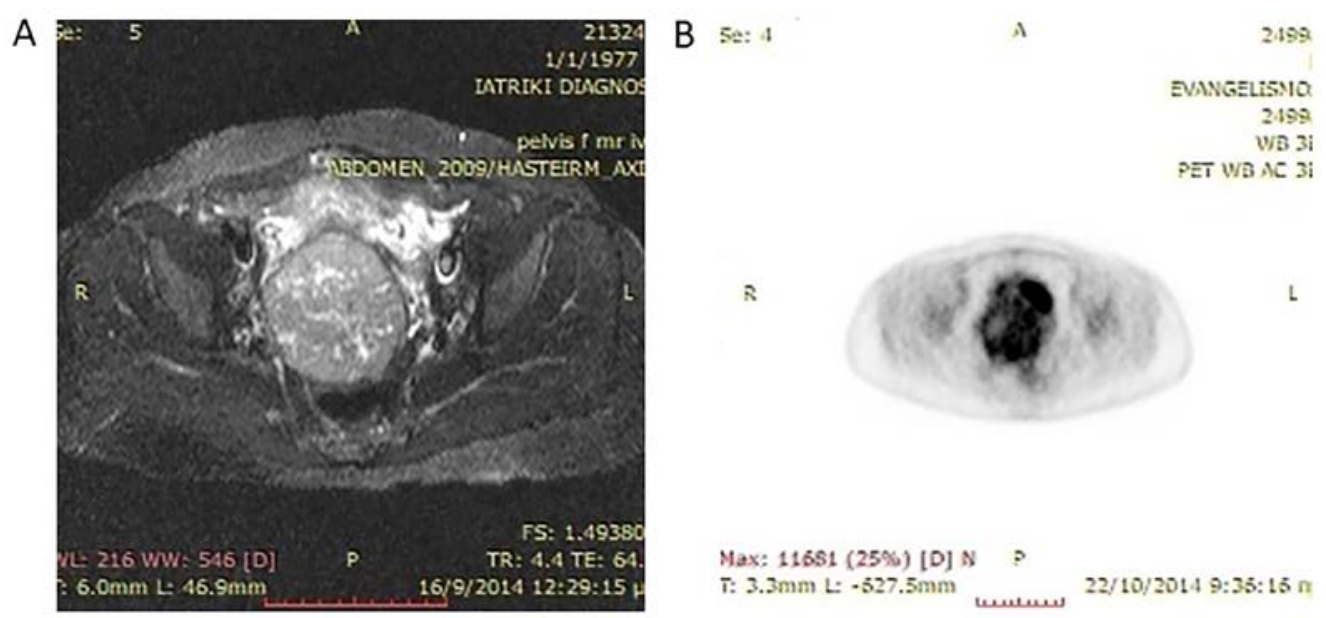

Figure 1. (A) MRI of abdomen and (B) PET-CT showing the tumor.
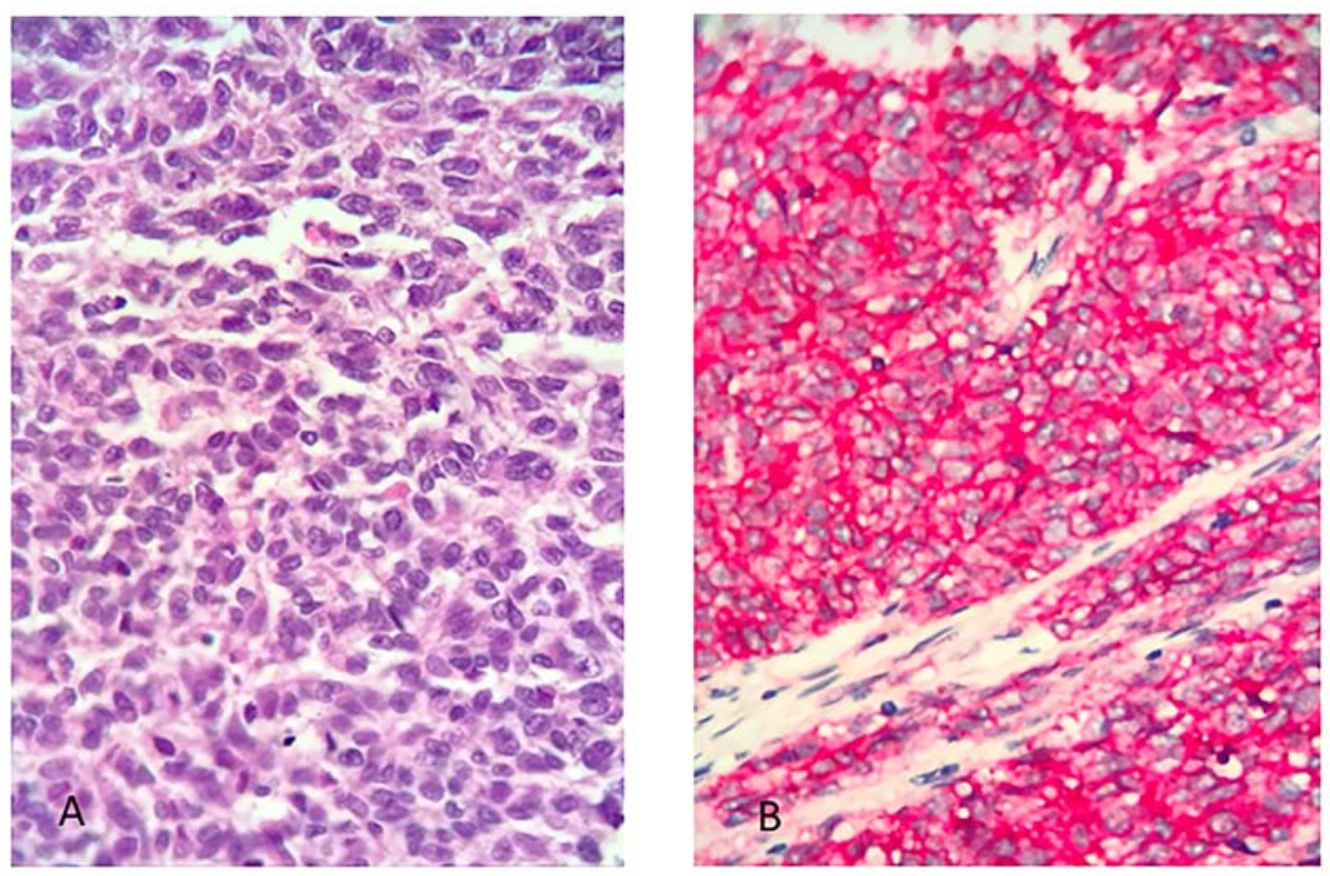

Figure 2. (A) Hematoxylin-eosin section of Ewing's sarcoma of cervix uteri, (x100 magnification). (B) Immunohistochemistry with CD99.

Clinical Staging of the disease according to FIGO stage system was IB2. Histopathology slides were reviewed by an independent pathologist who confirmed the diagnosis of Ewing's sarcoma/PNET.

The patient underwent radical hysterectomy with bilateral salpingoophorectomy and systematic pelvic lymphadenectomy. Pathology revealed a cervical tumor of $8.5 \times 7.7 \times 7 \mathrm{~cm}$, which invaded the cervical wall with no extension beyond it. Surgical margins were free of disease. Lymph nodes were free of metastasis. The overall immunomorphologic characteristics of the tumor favor the diagnosis of Ewing's sarcoma/PNET of the cervix (Fig. 2). The Ethics Committee of Alexandra Hospital (Athens, Greece) has approved this study and the patient has signed form of consent.

To further characterize this rare case, we performed genetic analyses to the tissue specimen. Fluoresence in situ hybridization (FISH) analysis with EWS break-apart kit revealed fusion of EWS gene (Fig. 3). RT-PCR detected the formation of EWS-FLI1 chimeric gene (Fig. 3).

The patient performed post-operative CT scans of her abdomen and her chest, which showed absence of residual disease or metastases. She received 6 cycles of adjuvant chemotherapy with VIDE (21): Vincristine $1.5 \mathrm{mg} / \mathrm{m}^{2}$ day 1, Ifosfamide $3 \mathrm{gr} / \mathrm{m}^{2}$ day 1-3, Doxorubicin $20 \mathrm{mg} / \mathrm{m}^{2}$ day 1-3, Etoposide $150 \mathrm{mg} / \mathrm{m}^{2}$ day $1-3$, Uromitexan day 1-3 (intravenous $20 \%$ of ifosfamide dose, per os $40 \%$ of ifosfamide dose 2 and $8 \mathrm{~h}$ after the infusion of ifosfamide), inj. GCSF 48MU day 7-14, and chemoprophylaxis with fluconazole $50 \mathrm{mgx} 1$ day 7-14, ciprofloxacin $250 \mathrm{mgx} 2$ day 7-14 and co-trimoxazole $480 \mathrm{mg}$ day 1-21. During the chemotherapy patient presented with Grade I nausea, Grade III anaemia, which was treated with Erythropoiesis stimulating agent and Grade I stomatitis, which was resolved after the administration of miconazole oral gel. After the completion 
Table I. Results of fluorescence in situ hybridization analysis.

\begin{tabular}{lrrccc}
\hline Cases & Ex.N & Br-Ap & NORMAL & Non Sp & \% ERWR1 \\
\hline Specimen & 213 & 129 & 35 & 49 & 60.5 \\
Control & 63 & 13 & 39 & 11 & 20.6 \\
\hline
\end{tabular}

Ex.N, total number of examined nuclei; Br-Ap, number of nuclei with Break-Apart signals; Non Sp, number of nuclei with non-specific signals.

of chemotherapy she received local pelvic radiotherapy (total dose 45 Gy). Forty two months after her diagnosis she remains free of disease.

Histopathology. Microscopic examination of the biopsy tissue specimen showed sheets of small to medium sized round primitive cells with 5 mitoses/10 HPF, harboring i) immunohistochemical positive markers: p16 and CD99 and ii) negative markers: SMA, desmin, S100, AE1/AE3, CD56, p63, LCA, CK18, chromogranin, favoring the diagnosis of Ewing's sarcoma/PNET. Histopathology slides were subsequently reviewed by an independent pathologist who described i) immunohistochemical positivity for Vimentin, Fli-1, MIC-2, EMA and NSE and ii) negativity for TdT, Desmin, CD7, CD79a, CD10, WT1, MyoD1, CD3, CD56, S-100, SMA, CEA, PR, ER, caldesmin, CK19, CK7, CK18, Chromogranin and Synaptophysin, confirming the diagnosis of Ewing's sarcoma/PNET.

Immunohistochemical staining of the tumor after total hysterectomy was positive for CD99, CD117 and vimentin, while it was negative for LCA, HMB-45, desmin, synaptophysin, chromogranin, keratin5/6 and keratin7. Surgical margins were free of disease. Lymph nodes were free of metastasis. The overall immunomorphologic characteristics of the tumor favor the diagnosis of Ewing's sarcoma/PNET of the cervix (Fig. 2).

\section{Genetics}

FISH. Formalin-fixed, paraffin-embedded biopsy blocks from the specimen were analyzed for the detection of translocations involving the EWSR gene at 22q12.2. A section of $4 \mu \mathrm{m}$ was cut from the selected block and applied to silinized slides. Additional serial sections from the representative block were stained with hematoxylin-eosin in order to confirm the presence of tumor cells and to choose the appropriate area for the hybridization procedures. A section from normal tissue was used as negative control. The slides, baked at $60^{\circ} \mathrm{C}$ for $4 \mathrm{~h}$, deparaffinized in 2 changes of fresh xylene for $10 \mathrm{~min}$ at RT, dehydrated for $5 \mathrm{~min}$ in $100 \%$ (twice), 90 and 70\% ethanol solutions and allowed to air-dry before application of the pretreatment kit (Zytolight FISH-Tissue kit; Zytovision GmbH, Bremerhaven, Germany) according to the manufacturer's instructions. For hybridization procedures, the FISH probe 'ZytoLight SPEC EWSR1 Dual Color Break Apart (Zytovision $\mathrm{GmbH}$ ) was used. Probe mixture was applied onto the areas of interest on the slides according to the manufacturer's instructions. Target areas were, afterwards, covered with glass coverslips and sealed with rubber cement. Two post-hybridization washes were performed in 2x SSC/0.3\% NP40. Slides were air-dried and counterstained using $10 \mu \mathrm{l}$ DAPI. The prepared slides were microscopically analyzed soon afterwards. Hybridization signals were counted by the use of a Zeiss Axioplan fluorescence microscope (Carl Zeiss AG, Oberkochen, Germany) equipped with the appropriate filter combination and the ISIS digital imaging system and software (MetaSystems Hard and Software GmbH, Altlußheim, Germany). The evaluation of FISH signals meet the following criteria: i) No overlapping cells are counted, ii) a probe is considered to be split (break-a-part) when the orange and the green signals are separated by two times distance greater than the size of one hybridization signal, iii) a sample is determined to be positive for EWSR gene translocation if the number of nuclei that carried the break apart signals exceeds the cutoff of the control sample.

$R T-q P C R$. Total RNA was extracted from FFPE sections using NucleoSpin total RNA FFPE Mini Kit (Macherey-Nagel, $\mathrm{GmbH}$ and Co., Düren, Germany), according to the manufacturer's instructions. Approximately $500 \mathrm{ng}$ of total RNA was reverse transcribed using the SuperScript II Reverse Transcriptase (Invitrogen; Thermo Fisher Scientific, Inc., Waltham, MA, USA) and random hexamers. cDNA was subjected to TaqMan qPCR analysis, for the detection of the EWS/Fli-1 fusion genes both type 1 and 2, using Platinum qPCR Supermix-UDG system (Invitrogen; Thermo Fisher Scientific, Inc.), with specific primers and PCR conditions as previously described $(22,23)$.

\section{Results}

Histopathology results favor the diagnosis of Ewing's sarcoma/PNET (Fig. 2). The results of FISH of the counted nuclei are presented to the Table I. According to Table I, $20 \%$ nuclei of the control sample appeared with break-a-part signals, whilst $60 \%$ of the specimen examined nuclei, carried split signals. These results confirm the presence EWSR1 gene translocations (Fig. 3).

The type 1 EWS/FLI fusion gene was detected in the examined specimen sections, which consists of the first seven exons of EWS joined to exons 6-9 of FLI1 and accounts for approximately $60 \%$ of reported Ewing's sarcoma cases (Fig. 3).

\section{Discussion}

Extraosseus Ewing's sarcoma is an uncommon malignancy of mesenchymal origin. Cervical Ewing's sarcoma is a rare entity with very few cases reported (Table II). Diagnosis of Ewing's/PNET sarcomas in many cases is a challenge. Especially, occurrence of Ewing's sarcoma in the female genital tract makes this diagnosis even more difficult. Herein we present a case of Ewing's sarcoma of the cervix in a pregnant woman.

In our case histopathology was complemented with genetic analysis to further confirm the diagnosis of Ewing's sarcoma. FISH and Real time PCR revealed the characteristic fusion gene EWS-FLI1. The presence of this fusion is associated with 
A

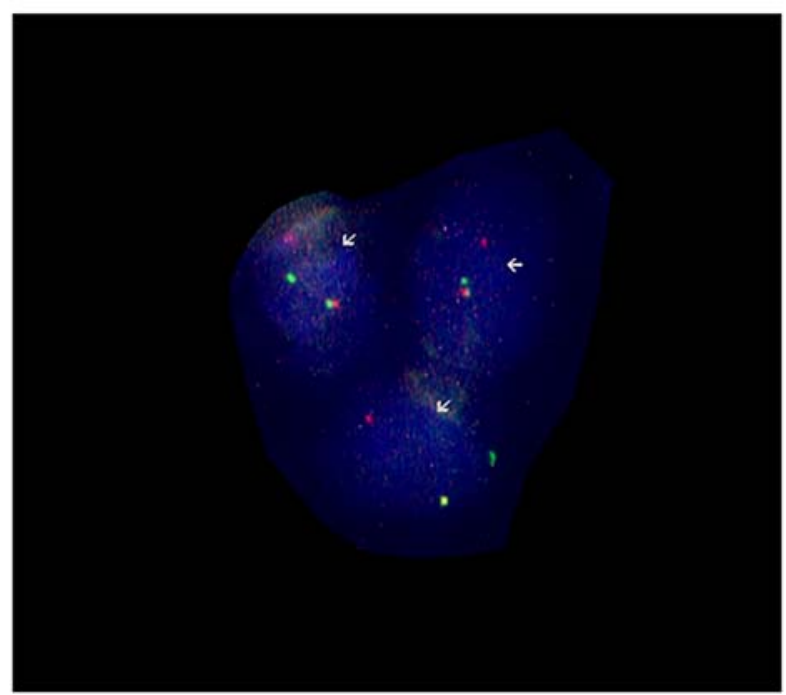

B

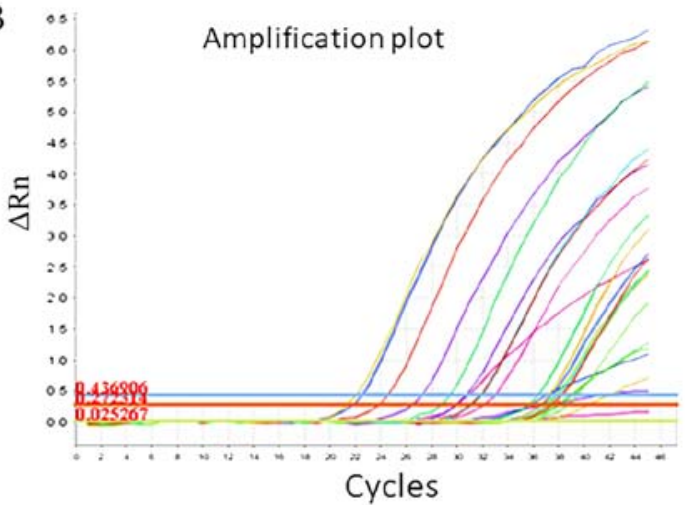

C

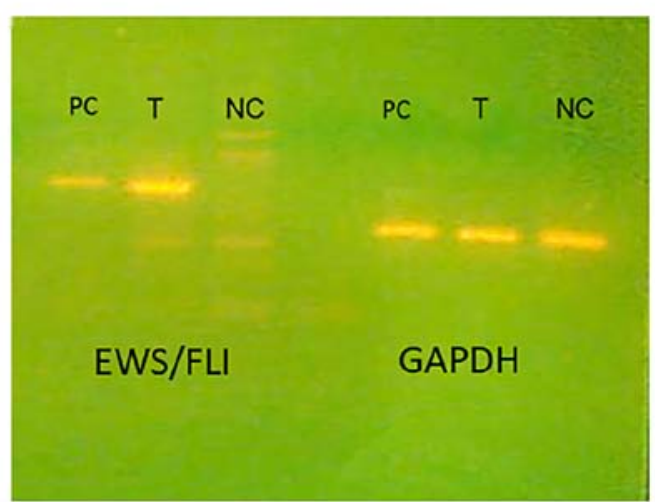

Figure 3. (A) FISH analysis with EWS break-a-part kit, Green indicated sequence mapping in 22q12.2 distal to EWSR1 gene and Red indicated sequence mapping in 22q12.1-q12.2 proximal to EWSR1 gene. Arrows illustrate cells with separation of the signal indicating fusion gene formation. The product of EWS/FLI chimeric gene of Type 1 was detected in the analyzed tissue by RT-qPCR where the (B) amplification plot and (C) gels are presented. PC, Positive control; T, Tumor sample; NC, Negative control; GAPDH gene. $\triangle$ Rn, normalized reporter value; FISH, fluoresence in situ hybridization.

a better prognosis and a more favorable clinical outcome (24). However, with the use of new chemotherapeutic regimens, non-type 1 fusion type carriers seem to share similar prognosis with patients harboring EWS/FLI1 chimeric gene. In the rare occasion of Ewing sarcomas of the female genital tract there are no data regarding molecular prognostication.

The patient remains free of disease after 42 months of follow up. Ewing sarcoma is an aggressive tumor with generally poor prognosis. Our case however, harbored some favorable clinicopathological characteristics. Mitotic index of her tumor was 5 mitoses/10 HPF indicating limited proliferation status. Several studies have shown that high mitotic index is an independent prognostic factor for sarcomas (25-28). Additionally, our patient presented with Figo Stage IB2 disease. Early tumor stage is another favorable prognostic factor confirmed in several reported studies $(26,27,29)$. Additionally, our patient was treated with radical hysterectomy and lymphadenectomy due to the large size of the tumor, in order to obtain clear margins (parametrium, upper vagina and sacro-uterine legaments) and avoid any lymph node involvement. However, it is difficult to support that lymphadenectomy has any added value to the prognosis of our patient.

Literature review revealed a few cases of cervical Ewing sarcoma which were treated with several chemotherapeutic regimens (Table II). The heterogeneity of the used regimens reflect the rarity of the disease and the evolution of multiagent chemotherapy for Ewing Sarcoma the last few decades (30-33). Our case is the only one, which was initially treated with radical hysterectomy and afterwards the patient has been treated with adjuvant VIDE, a chemotherapeutic option commonly used in extraosseus Ewing's sarcomas (21). Our patient was pregnant and her diagnosis was an incidental finding during her scheduled routine prenatal ultrasound. In the current literature, that extraosseus Ewing sarcoma diagnosis during pregnancy has been reported in 6 cases $(13,20)$. Only 5 of these cases received chemotherapy during pregnancy. The chemotherapeutic regimens used were: Doxorubicin-ifosfamide, actinomycine D-cyclophosphamide-vincristine-bleomycin-vincristine-doxorubicin, doxorubicin-cyclophosphamide-vincristine, VIDE scheme followed by Vincristine Adriamycin Cyclophosphamide (VAC). The latter combination caused the abortion due to oligohydramnion. Since our patient decided to end her pregnancy there was no clinical dilemma regarding the selection of the chemotherapeutic regimen. The biological mechanism by which pregnancy might be connected with Ewing sarcoma or cervical neoplasia is vague. However, there are data indicating that Ewing's sarcoma precursors are highly enriched in embryonic osteochondrogenic progenitors therefore providing clues to the histogenesis of Ewing's sarcoma (34). In our case the patient presented to our Department post-operatively and neoadjuvant schemes could not be administered to her.

To conclude, we present a case of cervical Ewing sarcoma, which was diagnosed during pregnancy. Diagnostic approach included both immunohistochemistry and genetic characterization of the tumor. This is a patient that was treated aggressively with tri-modality therapy according to Ewing's sarcoma experience and she is currently free of disease 3.5 years after her diagnosis. 


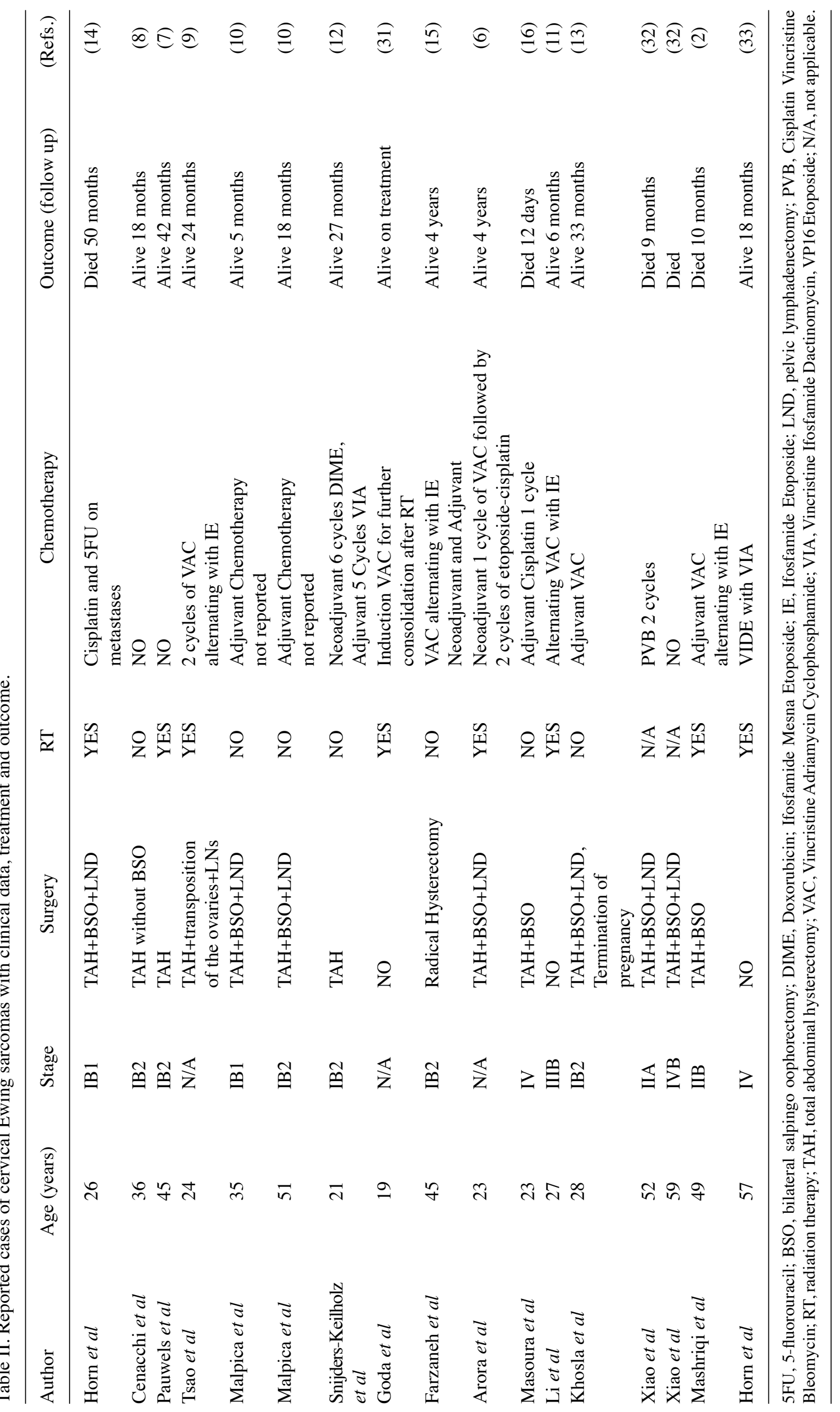




\section{Acknowledgements}

Not applicable.

\section{Funding}

No funding was received.

\section{Availability of data and materials}

The datasets used and/or analyzed during the current study are available from the corresponding author on reasonable request.

\section{Authors' contributions}

AK analyzed the patient, undertook analysis of the clinical, radiological and laboratory results and wrote the manuscript. GT also analyzed the patient, and analyzed the clinical, radiological and laboratory results. ML analyzed the patient, analyzed the clinical, radiological and laboratory results and drafted the manuscript. AP and LM analyzed and interpreted the molecular genetics and FISH tests and drafted the manuscript. GM and IP analyzed and interpreted the pathology tests and drafted the manuscript. NT analyzed, and operated on the patient, and contributed to the analysis of the clinical, radiological and laboratory results and drafted the manuscript Finally, AB analyzed the patient, analyzed the clinical, radiological and laboratory results and drafted the manuscript.

\section{Ethics approval and consent to participate}

The Ethics Committee of Alexandra Hospital has approved this study and the patient has signed form of consent for the analysis and publication of her data.

\section{Patient consent for publication}

The patient has provided consent for the analysis and publication of her data.

\section{Competing interests}

The authors declare that they have no competing interests.

\section{References}

1. Fadare O: Uncommon sarcomas of the uterine cervix: A review of selected entities. Diagn Pathol 1: 30, 2006.

2. Mashriqi N, Gujjarlapudi JK, Sidhu J,Zur M and Yalamanchili M Ewing's sarcoma of the cervix, a diagnostic dilemma: A case report and review of the literature. J Med Case Rep 9: 255, 2015

3. Applebaum MA, Worch J, Matthay KK, Goldsby R, Neuhaus J, West DC and Dubois SG: Clinical features and outcomes in patients with extraskeletal Ewing sarcoma. Cancer 117: 3027-3032, 2011.

4. de Alava E and Gerald WL: Molecular biology of the Ewing's sarcoma/primitive neuroectodermal tumor family. J Clin Oncol 18: 204-213, 2000

5. Sato S, Yajima A, Kimura N, Namiki T, Furuhashi $\mathrm{N}$ and Sakuma H: Peripheral neuroepithelioma (peripheral primitive neuroectodermal tumor) of the uterine cervix. Tohoku J Exp Med 180: 187-195, 1996.

6. Arora N, Kalra A, Kausar H, Ghosh TK and Majumdar A: Primitive neuroectodermal tumour of uterine cervix-a diagnostic and therapeutic dilemma. J Obstet Gynaecol 32: 711-713, 2012.
7. Pauwels P, Ambros P, Hattinger C, Lammens M, Dal Cin P, Ribot J Struyk A and van den Berghe H: Peripheral primitive neuroectodermal tumour of the cervix. Virchows Arch 436: 68-73, 2000.

8. Cenacchi G, Pasquinelli G, Montanaro L, Cerasoli S, Vici M, Bisceglia M, Giangaspero F, Martinelli GN and Derenzini M: Primary endocervical extraosseous Ewing's sarcoma/PNET. Int J Gynecol Pathol 17: 83-88, 1998.

9. Tsao AS, Roth LM, Sandler A and Hurteau JA: Cervical primitive neuroectodermal tumor. Gynecol Oncol 83: 138-142, 2001.

10. Malpica A and Moran CA: Primitive neuroectodermal tumor of the cervix: A clinicopathologic and immunohistochemical study of two cases. Ann Diagn Pathol 6: 281-287, 2002.

11. Li B, Ouyang L, Han X, Zhou Y, Tong X, Zhang S and Zhang Q: Primary primitive neuroectodermal tumor of the cervix. Onco Targets Ther 6: 707-711, 2013.

12. Snijders-Keilholz A, Ewing P, Seynaeve $C$ and Burger CW: Primitive neuroectodermal tumor of the cervix uteri: A case report-changing concepts in therapy. Gynecol Oncol 98: 516-519, 2005.

13. Khosla D, Rai B, Patel FD, Sreedharanunni S, Dey P and Sharma SC: Primitive neuroectodermal tumor of the uterine cervix diagnosed during pregnancy: A rare case with review of literature. J Obstet Gynaecol Res 40: 878-882, 2014.

14. Horn LC, Fischer U and Bilek K: Primitive neuroectodermal tumor of the cervix uteri. A case report. Gen Diagn Pathol 142: 227-230, 1997.

15. Farzaneh F, Rezvani H, Boroujeni PT and Rahimi F: Primitive neuroectodermal tumor of the cervix: A case report. J Med Case Rep 5: 489, 2011.

16. Masoura S, Kourtis A, Kalogiannidis I, Kotoula V, Anagnostou E, Angelidou $S$ and Agorastos T: Primary primitive neuroectodermal tumor of the cervix confirmed with molecular analysis in a 23-year-old woman: A case report. Pathol Res Pract 208: 245-249, 2012.

17. Wang X, Gao Y, Xu Y, Liu Y and Qu P: Primary primitive neuroectodermal tumor of the cervix: A report of two cases and review of the literature. Mol Clin Oncol 6: 697-700, 2017.

18. ESMO/European Sarcoma Network Working Group: Soft tissue and visceral sarcomas: ESMO Clinical Practice Guidelines for diagnosis, treatment and follow-up. Ann Oncol 25 (Suppl 3): iii102-iii112, 2014

19. Demetri GD, Baker LH, Beech D, Benjamin R, Casper ES, Conrad EU III, DeLaney TF, Ettinger DS, Heslin MJ, Hutchinson RJ, et al: Soft tissue sarcoma clinical practice guidelines in oncology. J Natl Compr Canc Netw 3: 158-194, 2005.

20. Schur S, Wild J, Amann G, Kostler W, Langer M and Brodowicz T: Sarcoma of the ewing family in pregnancy: A case report of intrauterine fetal death after induction of chemotherapy. Case Rep Oncol 5: 633-638, 2012.

21. Strauss SJ, McTiernan A, Driver D, Hall-Craggs M, Sandison A, Cassoni AM, Kilby A, Michelagnoli M, Pringle J, Cobb J, et al: Single center experience of a new intensive induction therapy for ewing's family of tumors: Feasibility, toxicity, and stem cell mobilization properties. J Clin Oncol 21: 2974-2981, 2003.

22. Lewis TB, Coffin CM and Bernard PS: Differentiating Ewing's sarcoma from other round blue cell tumors using a RT-PCR translocation panel on formalin-fixed paraffin-embedded tissues. Mod Pathol 20: 397-404, 2007.

23. Peter M, Gilbert E and Delattre O: A multiplex real-time pcr assay for the detection of gene fusions observed in solid tumors. Lab Invest 81: 905-912, 2001.

24. de Alava E, Panizo A, Antonescu CR, Huvos AG, Pardo-Mindán FJ, Barr FG and Ladanyi M: Association of EWS-FLI1 type 1 fusion with lower proliferative rate in Ewing's sarcoma. Am J Pathol 156: 849-855, 2000.

25. Feng W, Malpica A, Robboy SJ, Gudlaugsson E, Hua K, Zhou X and Baak JP: Prognostic value of the diagnostic criteria distinguishing endometrial stromal sarcoma, low grade from undifferentiated endometrial sarcoma, 2 entities within the invasive endometrial stromal neoplasia family. Int $\mathbf{J}$ Gynecol Pathol 32: 299-306, 2013.

26. Lusby K, Savannah KB, Demicco EG, Zhang Y, Ghadimi MP, Young ED, Colombo C, Lam R, Dogan TE, Hornick JL, et al: Uterine leiomyosarcoma management, outcome, and associated molecular biomarkers: A single institution's experience. Ann Surg Oncol 20: 2364-2372, 2013.

27. Pautier P, Genestie C, Rey A, Morice P, Roche B, Lhommé C, Haie-Meder C and Duvillard P: Analysis of clinicopathologic prognostic factors for 157 uterine sarcomas and evaluation of a grading score validated for soft tissue sarcoma. Cancer 88: $1425-1431,2000$ 
28. Gremel G, Liew M, Hamzei F, Hardell E, Selling J, Ghaderi M, Stemme S, Pontén F and Carlson JW: A prognosis based classification of undifferentiated uterine sarcomas: Identification of mitotic index, hormone receptors and YWHAE-FAM22 translocation status as predictors of survival. Int J Cancer 136: $1608-1618,2015$.

29. Feng W, Malpica A, Skaland I, Gudlaugsson E, Robboy SJ, Dalen I, Hua K,Zhou X and Baak JP: Can proliferation biomarkers reliably predict recurrence in World Health Organization 2003 defined endometrial stromal sarcoma, low grade? PLoS One 8: e75899, 2013.

30. Grier HE, Krailo MD, Tarbell NJ,Link MP, Fryer CJ,Pritchard DJ, Gebhardt MC, Dickman PS, Perlman EJ, Meyers PA, et al: Addition of ifosfamide and etoposide to standard chemotherapy for Ewing's sarcoma and primitive neuroectodermal tumor of bone. N Engl J Med 348: 694-701, 2003.

31. Goda J, Mayur BN, Pramod PK and Udayan K: Primitive neuroectodermal tumour of the Cervix: A rare entity. Int J Radiol 6 , 2006.
32. Xiao C, Zhao J, Guo P, Wang D, Zhao D, Ren T, Yang J, Shen K, Lang J, Xiang Y and Cui Q: Clinical analysis of primary primitive neuroectodermal tumors in the female genital tract. Int $\mathrm{J}$ Gynecol Cancer 24: 404-409, 2014.

33. Bílek O, Holánek M, Zvaríková M, Fabian P, Robešová $\mathrm{B}$, Procházková M and Adámková Krákorová D: Extraoseus ewings sarcoma, primary affection of uterine cervix-case report. Klin Onkol 28: 284-287, 2015 (In Czech).

34. Tanaka M, Yamazaki Y, Kanno Y, Igarashi K, Aisaki K, Kanno J and Nakamura T: Ewing's sarcoma precursors are highly enriched in embryonic osteochondrogenic progenitors. J Clin Invest 124: 3061-3074, 2014.

(c) (i) () $($ This work is licensed under a Creative Commons International (CC BY-NC-ND 4.0) License. 